\title{
RENDIMENTO E VARIEDADE DE PRODUTOS NO DESDOBRO DE TORAS DE Eucalyptus spp.
}

\author{
Douglas Edson Carvalho ${ }^{*}$, Márcio Pereira da Rocha ${ }^{2}$, Romano Timofeiczyk Junior ${ }^{2}$, Ricardo Jorge Klitzke ${ }^{2}$ \\ 1 Programa de Pós-graduação em Engenharia Florestal, Universidade Federal do Paraná, 80201-170, Curitiba, Brasil. \\ 2 Departamento de Engenharia e Tecnologia Florestal, Universidade Federal do Paraná, 80201-170, Curitiba, Brasil.
}

*E-mail: douglasedsoncarvalho@gmail.com

\section{RESUMO}

O estudo foi realizado em uma serraria com o objetivo de avaliar a conicidade, o rendimento, a variedade e as quantidades de produtos oriundos do desdobro de toras de Eucalyptus spp., serradas por meio de dois modelos de desdobro, em duas classes diamétricas. Foram utilizadas toras de Eucalyptus spp., as quais foram separadas em uma classe de diâmetro médio de $33,0 \mathrm{~cm}$ e uma classe de diâmetro médio de $47,0 \mathrm{~cm}$. Para a classe de menor diâmetro foi aplicado o modelo de desdobro do tipo tangencial alternado e para a classe de maior diâmetro foi aplicado o modelo de desdobro do tipo radial. A partir do processamento das toras, os produtos obtidos foram classificados e quantificados, obtendo o rendimento de cada modelo de desdobro. Quanto ao parâmetro conicidade, os resultados representaram toras de boa qualidade, variando de $0,92 \mathrm{~cm} / \mathrm{m}$ na menor classe diamétrica a $1,42 \mathrm{~cm} / \mathrm{m}$ na maior classe diamétrica. $O$ modelo de desdobro radial, além de apresentar maior rendimento $(53,43 \%)$ em relação ao modelo de desdobro tangencial alternado $(47,08 \%)$, apresentou maior percentual do produto considerado de maior valor agregado (viga). A escolha do modelo de desdobro adequado para cada classe diamétrica é um método indicado para reduzir as perdas e maximizar os rendimentos em serrarias.

Palavras-chave: Madeira serrada. Rentabilidade. Sustentabilidade. Qualidade.

\section{Introdução}

Entre as alternativas de espécies madeireiras utilizadas e oriundas de sistemas de manejo controlados, Müller et al. [1] (2014) citam a utilização de madeira serrada de eucalipto, que embora ainda com produção em pequena escala, acredita-se que em curto espaço de tempo a situação será revertida, principalmente pelo aperfeiçoamento de técnicas de desdobro, secagem e beneficiamento da matéria-prima. Este fato, somado ao aproveitamento adequado da madeira, torna o gênero Eucalyptus spp. uma boa alternativa com potencial de uso sustentável em larga escala.

Alguns fatores inerentes à madeira são fundamentais para obter qualidade e rendimento adequado das toras, entre eles citamse os fatores intrínsecos à espécie como tensões de crescimento, e fatores extrínsecos como conicidade e curvatura da tora (Ferreira et al. [2] 2004).

Corroborando com isso, Valério et al. [3] (2007) mencionam a conicidade como um desses fatores, uma vez que esse parâmetro está relacionado com a diminuição do diâmetro do tronco, que ocorre da base para a copa da árvore. Este parâmetro pode resultar em desperdícios elevados no momento de ajuste das dimensões das toras e, consequentemente, em perdas de rendimento.

Além disto, Juizo et al. [4] (2014) citam como fator preponderante para obtenção de rendimento satisfatório e qualidade adequada da madeira serrada, o uso do modelo de desdobro adequado à matéria-prima. Vital [5] (2008) também menciona que o modelo de corte utilizado altera o rendimento e a qualidade, assim como a eficiência da serraria.

Sendo assim, o uso de modelos de corte apropriados a cada tipo de tora e a diversidade de produtos obtidos no processo de desdobro são formas de aumentar o rendimento em madeira serrada, tornando o processo mais rentável e sustentável. Consequentemente, o tipo de desdobro aplicado pode gerar peças mais estáveis dimensionalmente, agregando qualidade ao produto serrado.

Deste modo, a aplicação de métodos de corte adequado à classe diamétrica ideal para cada produto objetiva agregar qualidade e rentabilidade ao processo produtivo, reduzindo custos 
de modo que torne as empresas mais competitivas e atuantes no mercado.

Neste contexto, o estudo objetivou avaliar a conicidade, o rendimento e os tipos de produtos provenientes de dois modelos de desdobro de toras de Eucalyptus spp.

\section{Materiais e métodos}

\subsection{Local de estudo e volume de toras}

O estudo foi conduzido em uma serraria de médio porte localizada na região metropolitana de Curitiba-PR. Para o desdobro principal de toras utilizou-se uma serra de fita vertical simples com volante de $1.350 \mathrm{~mm}$ de diâmetro e uma serra de fita vertical simples com volante de $1.100 \mathrm{~mm}$ de diâmetro. Para resserragem da madeira utilizou-se uma serra de fita vertical simples com volante de $800 \mathrm{~mm}$ de diâmetro, uma serra circular múltipla de um eixo, uma serra circular refiladeira e três serras circulares destopadeiras.

Foram utilizadas 203 toras de Eucalyptus spp., as quais foram separadas em toras de diâmetro médio de $33,0 \mathrm{~cm}$ e 47,0 $\mathrm{cm}$, originando duas classes diamétricas, sem distinção de espécie. Após serem separadas em classes, foram realizadas medições de comprimento e, na ponta fina e na ponta grossa, a circunferência (mm) das toras com auxílio de fita métrica e transformada em diâmetro $(\mathrm{cm})$ para posterior cálculo de volume conforme adaptação da equação 1 de Smalian:

Vtora $=\frac{\pi}{40000} *\left(\frac{D 1+D 2}{2}\right)^{2} * C$

Em que:

Vtora: volume da tora $\left(\mathrm{m}^{3}\right)$;

D1: diâmetro da ponta fina $(\mathrm{cm})$;

D2: diâmetro na ponta grossa $(\mathrm{cm})$;

$C$ : comprimento da tora $(\mathrm{m})$.

\subsection{Conicidade das toras}

Para o cálculo da conicidade das toras foi utilizada a equação 2, conforme Scanavaca Jr. e Garcia [6] (2003):

Conic $=\frac{D 2-D 1}{C}$

em que:

Conic: conicidade da tora $(\mathrm{cm} / \mathrm{m})$;

\subsection{Modelos de desdobro}

As duas classes diamétricas foram submetidas a dois modelos de desdobro. Para toras de menor diâmetro $(33,0 \mathrm{~cm})$ foi aplicado o modelo de desdobro do tipo tangencial alternado (Figura 1A) e para toras de maior diâmetro $(47,0 \mathrm{~cm})$ foi aplicado o modelo de desdobro do tipo radial (Figura 1B).
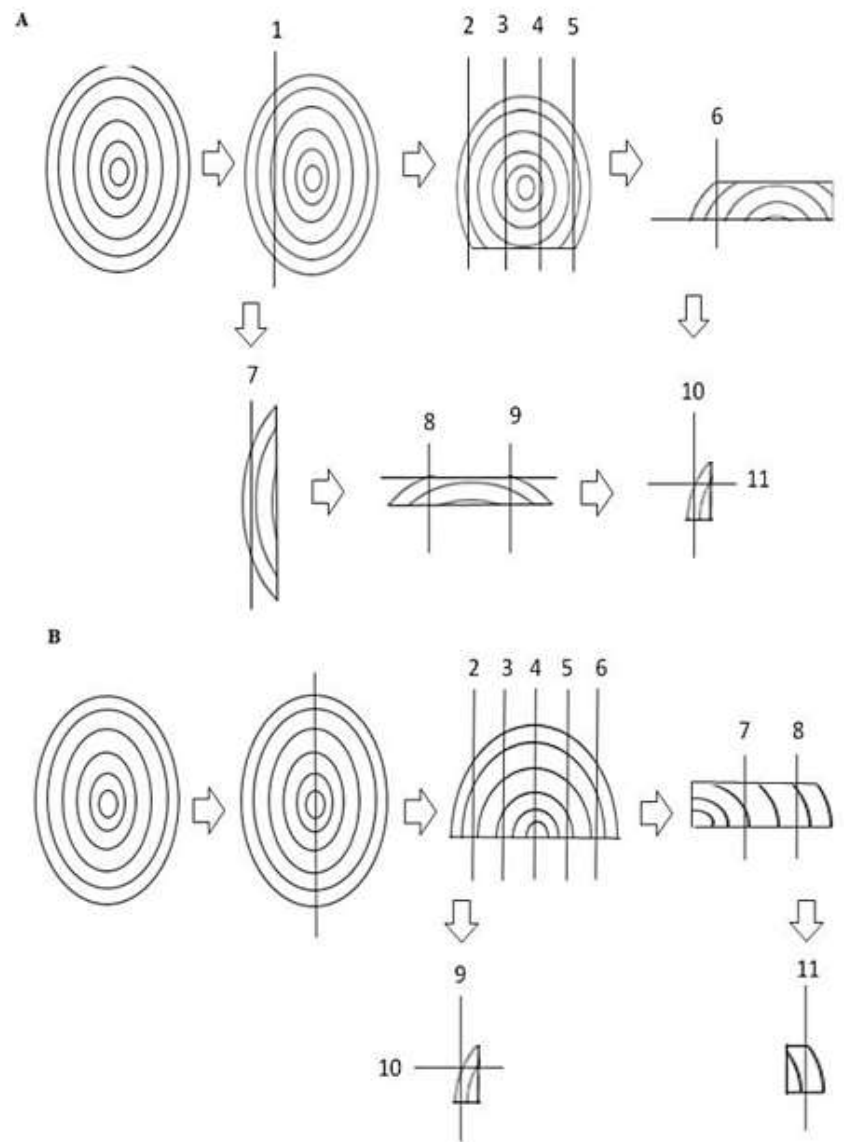

Figura 1A. Modelo de desdobro tipo tangencial alternado aplicado em toras de eucalipto para a classe de diâmetro de $33 \mathrm{~cm}$. 1B. Processo de desdobro radial aplicado em toras de eucalipto para a classe de diâmetro de $47 \mathrm{~cm}$.

\subsection{Volume de madeira serrada e rendimento}

Para determinação do volume de madeira serrada foram tomadas medidas das espessuras, larguras e comprimento das peças individualmente e, posteriormente, o somatório das mesmas, conforme Equações 3 e 4, adaptadas de Rocha [7] (2002): 


$$
\begin{aligned}
& V p e c ̧ a=L p^{*} E p^{*} C p \\
& \text { V.m.s. }=\sum V \text { Veça }
\end{aligned}
$$

em que:

Vpeça: volume de cada peça $\left(\mathrm{m}^{3}\right)$;

$L p$ : largura da peça $(\mathrm{m})$;

Ep: espessura da peça $(\mathrm{m})$;

$C p$ : comprimento da peça $(\mathrm{m})$;

V.m.s.: volume de madeira serrada $\left(\mathrm{m}^{3}\right)$.

Foram aferidas as larguras e as espessuras em três posições com um paquímetro digital, sendo duas medições a $20 \mathrm{~cm}$ das extremidades e a terceira medição na posição central da peça, e o comprimento medido de topo a topo da peça com auxílio de uma trena. Para cálculo de rendimento foi utilizada a Equação 5, citada por Rocha [7] (2002).

$$
\operatorname{Rnd}(\%)=\frac{\text { V.m.s. }}{\text { Vtora }} * 100
$$

em que:

Rnd (\%): Rendimento em percentagem;

VTora: Volume da tora $\left(\mathrm{m}^{3}\right)$.

Posteriormente à obtenção da madeira serrada de cada modelo de desdobro, cada peça foi classificada conforme a especificação de largura e espessura, respectivamente, e comprimento variável, resultando nos produtos conforme a Tabela 1.

Tabela 1. Produtos obtidos em dois modelos de desdobro para toras de Eucalyptus spp. e suas respectivas dimensões.

\begin{tabular}{lccc} 
Produto & $\begin{array}{c}\text { Espessur } \\
\mathbf{a}(\mathbf{m m})\end{array}$ & $\begin{array}{c}\text { Largura } \\
(\mathbf{m m})\end{array}$ & $\begin{array}{c}\text { Compriment } \\
\mathbf{0}(\mathbf{m m})\end{array}$ \\
\hline Viga 11 & 50 & 110 & 2500 a 5000 \\
Viga 15 & 50 & 150 & 2500 a 5000 \\
Caibro & 50 & 50 & 2500 a 5000 \\
$\begin{array}{l}\text { Aproveitament } \\
\text { o }\end{array}$ & 25 & 50 & $<2500$ \\
Ripamento & 25 & 50 & 2500 a 5000 \\
Moveleiro & $25 / 35$ & $75 / 110 / 150$ & 1000 a 5000 \\
\hline
\end{tabular}

Após o somatório de volume de cada material, obteve-se o percentual que cada modelo de desdobro originou de cada produto. Ressalta-se que o produto de maior valor agregado é a viga, voltada para a construção civil. Deste modo, a madeira que não originava viga foi destinada à produção de outros materiais (aproveitamento, caibro, moveleiro, ripamento), denominados de produtos secundários, sempre com o intuito de maximizar o aproveitamento da tora.

\subsection{Análise dos dados}

Previamente à análise de variância (Anova) foi realizado o teste de Skewness e Kurtosis para avaliar a normalidade dos dados. A Anova foi realizada para identificar diferença significativa a 95\% de probabilidade para os fatores conicidade e volume médio de toras. Identificada a diferença estatística, procedeu-se com a comparação de médias por meio do teste de Tukey a 95\% de probabilidade. Todas as análises estatísticas foram realizadas com o auxílio do software Statgraphics Centurion XV. II.

\section{Resultados e discussões \\ 3.1 Conicidade e rendimento}

Na Tabela 2 são apresentados os valores do diâmetro médio das toras e os resultados da conicidade, volume médio por tora, volume médio de madeira serrada por tora e rendimento de cada modelo de desdobro.

Tabela 2. Diâmetro médio das toras (D.m.t.), conicidade (Con.), volume médio das

\begin{tabular}{|c|c|c|c|c|c|}
\hline Modelo & $\begin{array}{l}\text { D.m.t. } \\
\text { (cm) }\end{array}$ & $\begin{array}{c}\text { Con. } \\
(\mathrm{cm} / \mathrm{m})\end{array}$ & $\begin{array}{l}\text { V.m.t. } \\
\left(\mathbf{m}^{3}\right)\end{array}$ & $\begin{array}{c}\text { V.m.s.t. } \\
\left(\mathbf{m}^{3}\right)\end{array}$ & $\underset{(\%)}{R}$ \\
\hline $\begin{array}{l}\text { Tangencial } \\
\text { alternado }\end{array}$ & 33,0 & $\begin{array}{c}0,92 \\
(39,97 \%) \\
\text { B }\end{array}$ & $\begin{array}{c}0,4135 \\
(31,03 \%) \\
\text { B }\end{array}$ & 0,1977 & 47,08 \\
\hline Radial & 47,0 & $\begin{array}{c}1,48 \\
(38,89 \%) \\
\mathrm{A}\end{array}$ & $\begin{array}{c}0,6836 \\
(30,13 \%) \\
\mathrm{A}\end{array}$ & 0,3652 & 53,43 \\
\hline Média & - & 1,10 & 0,4987 & - & 49,82 \\
\hline
\end{tabular}
toras (V.m.t.), volume médio de madeira serrada por tora (V.m.s.t.) e rendimento médio $(\mathrm{R})$ da madeira de eucalipto desdobradas em dois modelos de desdobro

As médias seguidas na coluna por uma mesma letra maiúscula não diferem estatisticamente entre si pelo teste de Tukey em nível de $95 \%$ de probabilidade. Valores entre parênteses representam o coeficiente de variação.

Observa-se na Tabela 2 que a conicidade para as toras de maiores diâmetros foi $60,87 \%$ superior à conicidade das toras de menores diâmetros. Porém, é importante observar que tanto as toras de maiores diâmetros quanto as toras de menores diâmetros apresentaram baixa conicidade, podendo serem consideradas toras de boa forma para essa característica.

Embora o resultado mais elevado para conicidade média tenha sido constatado para as toras do modelo radial, é possível que esta característica tenha afetado pouco o rendimento médio 
neste tipo de desdobro, uma vez que o rendimento médio para este modelo foi maior que o rendimento médio obtido para o modelo tangencial alternado, o qual apresentou conicidade média menor.

A maior conicidade obtida para as toras do modelo de desdobro radial foi pela posição das mesmas ao longo do fuste, sendo que a maioria delas procedeu das primeiras e segundas toras da árvore, localizadas na base do fuste, em que a redução do diâmetro ocorre de maneira mais abrupta, ou seja, há maior afilamento do fuste em comparação à parte mediana da árvore de onde foram provenientes as toras do modelo tangencial alternado. Corroborando com tal afirmação, Husch, Miller e Beers [8] (1982) relatam que a forma das árvores pode ser descrita geometricamente, de modo geral, como um neiloide na base, um paraboloide no meio e um cone na extremidade final.

Concordando com esta disparidade de conicidade entre toras da base do fuste e as restantes, Gonçalves et al. [9] (2010), estudando a qualidade da madeira de híbrido clonal de Eucalyptus urophylla $x$ Eucalyptus grandis, obtiveram conicidade maior em toras da base em relação às demais.

Apesar desta diferença de conicidade, conforme a Norma para Classificação de Toras de Madeira de Folhosas [10] (IBDF, 1984), valores de conicidade inferiores a $3 \mathrm{~cm} / \mathrm{m}$ representam toras de boa qualidade, o que comprova a utilização de toras com qualidades apropriadas para a finalidade de madeira serrada, pois apresentaram conicidade abaixo do valor normativo. Estes resultados ganham destaque por permitir bom desempenho no aproveitamento das toras no momento do desdobro das mesmas, evitando operações desnecessárias para alinhamento da tora, que acarreta em peças não aproveitáveis, gerando subprodutos.

Em pesquisa com Eucalyptus cloeziana, Del Menezzi e Nahuz [11] (2001) obtiveram valores de conicidade de $0,96 \mathrm{~cm} / \mathrm{m}$ e $0,66 \mathrm{~cm} / \mathrm{m}$ na primeira e segunda tora, respectivamente, valores próximos aos encontrados para as toras de menor diâmetro deste estudo, porém como citado pelos autores, foi observada diferença neste parâmetro entre as toras de base e toras da porção mediana. Já Lopes et al. [12] (2004), avaliando árvores matrizes de Eucalyptus grandis, obtiveram valor médio de $1,41 \mathrm{~cm} / \mathrm{m}$, semelhante ao resultado encontrado para as toras de diâmetro mais elevado deste estudo, o que favorece o indicativo de toras com características aceitáveis neste parâmetro.

O rendimento médio maior para o desdobro radial pode ser explicado pelo tipo de desdobro aplicado como também pelos diâmetros maiores das toras deste modelo, assim como resultado obtido por Rocha [13] (2000), o qual descreve que o resultado de menor rendimento com toras de menores diâmetros é considerado normal em serrarias.
O mesmo comportamento já foi observado por Vianna Neto [14] (1984), em que o autor afirma que o incremento no diâmetro das toras aumenta o rendimento de madeira serrada e apresenta influência direta neste parâmetro.

Semelhante às pesquisas citadas anteriormente, Santos [15] (2010), estudando o rendimento do desdobro de toras de $E$. camaldulensis com 11 anos de idade, obtiveram resultados de $35,33 \%$ para a classe diamétrica de 14 a $19,9 \mathrm{~cm}$, e de $40,13 \%$ de rendimento para $E$. urophylla com dez anos de idade e diâmetros que variam entre 20 e $25 \mathrm{~cm}$, o que enfatiza a relação entre acréscimo de diâmetro com o aumento de rendimento. Assim, o incremento em diâmetro é, de fato, determinante no aumento do rendimento em madeira serrada, conferindo melhor aproveitamento da tora.

Em estudo elaborado por Biasi e Rocha [16] (2007), avaliando o rendimento de espécies da Amazônia, cedrinho (Erisma uncinatum), cambará (Qualea albiflora) e itaúba (Mezilaurus itauba), com toras de 31 a $70 \mathrm{~cm}$ de diâmetro, os autores obtiveram rendimento médio de 59,83\%, 62,63\% e $53,90 \%$ para as três espécies, respectivamente. Estes valores foram superiores aos obtidos no presente estudo, porém os autores trabalharam somente com toras de comprimentos igual a $2,75 \mathrm{~m}$, menores do que os do presente estudo, favorecendo o desdobro e maximizando o rendimento. Todavia, os autores citam fatores que limitam o suprimento de matéria-prima, como o baixo número de espécies comercializáveis e as distâncias entre floresta e indústria, que acabam onerando o preço da matéria-prima.

Melo et al. [17] (2016), em estudo com a madeira de Qualea sp, avaliando o desdobro de toras de 4,00 e 6,00 m, obtiveram rendimento máximo de $57 \%$ em toras de grande diâmetro (>75), porém em diâmetros menores $(55$ a $75 \mathrm{~cm})$ o rendimento reduziu para $49 \%$ e os autores enfatizam que fatores relacionados a ocos e insetos afetam diretamente no rendimento obtido.

Esses parâmetros servem de base comparativa para a utilização da madeira serrada de eucalipto em substituição às espécies nativas, as quais, além destas limitações citadas, ainda apresentam crescimento lento se comparado à madeira de florestas plantadas, em especial as do gênero Eucalyptus ssp.

\subsection{Produtos obtidos no desdobro}

A Figura 2 apresenta o percentual gerado de produtos nos dois modelos de desdobro avaliados. 
Figura 2 - Quantidade percentual de produtos obtidos de dois modelos de desdobro de madeira de eucalipto.

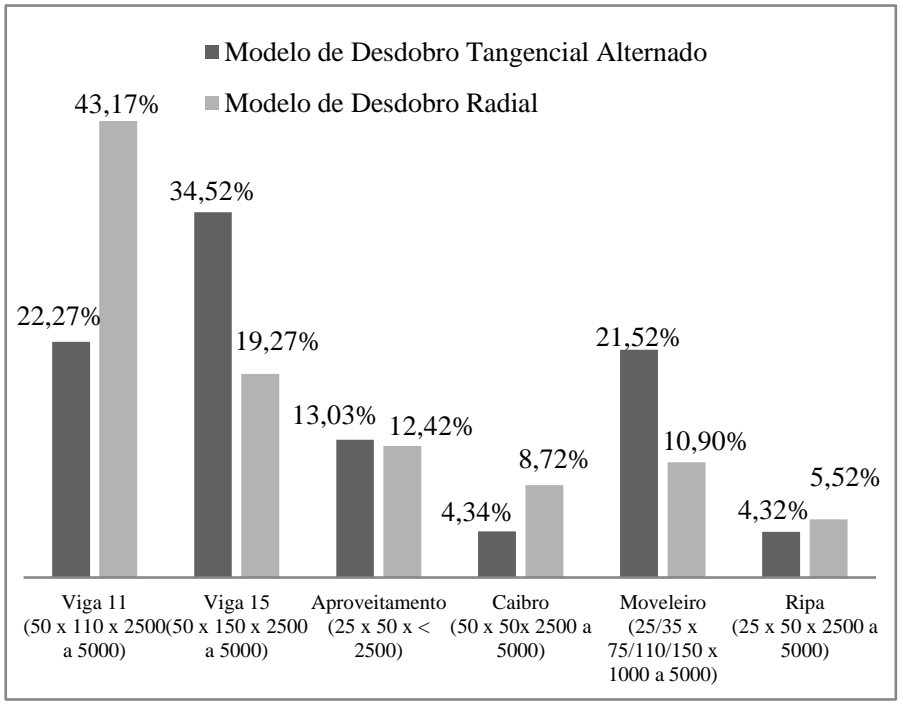

Em que: Entre parênteses as dimensões de cada produto, espessura, largura e comprimento, respectivamente (mm). Fonte: o autor (2018).

Somando os resultados percentuais para os produtos principais da empresa (viga 11 e viga 15 ), obtiveram-se $56,79 \%$ do total de madeira serrada para o modelo de desdobro tangencial alternado. Já utilizando o modelo de desdobro radial, além de apresentar peças mais estáveis dimensionalmente, o somatório destes produtos atingiu $62,44 \%$.

Este fato evidencia que toras com diâmetros elevados e de boa qualidade, como as utilizadas neste estudo, atrelado ao modelo de desdobro ideal para cada classe diamétrica resultam em elevados rendimentos de produtos de maior valor agregado.

Em estudo realizado por Garcia [18] (2013) com 895 toras da espécie Qualea albiflora (cambará) em serraria no município de Alta Floresta - Mato Grosso, com diâmetros que variam de 33 $\mathrm{cm}$ a $80 \mathrm{~cm}$, o autor obteve rendimento médio de 48,90\% para madeira de vigamento. Os resultados citados por Garcia (2013) estão abaixo dos obtidos no presente estudo e esta rentabilidade é fator essencial em termos de aproveitamento sustentável e adequado da madeira.

Segundo Monteiro et al. [19] (2013), as informações sobre quais subprodutos estão sendo produzidos, assim como a sua porcentagem, são fatores relevantes para a melhor gestão da indústria e a redução dos impactos ambientais. Deste modo, em relação à madeira de ripa, houve acréscimo de 1,2\% de rendimento no desdobro tangencial alternado para o desdobro radial, pelo aumento de refilo do vigamento, o que originava mais madeira para ser reaproveitada como ripa.
Quanto à madeira de aproveitamento ocorreu uma pequena redução de $0,61 \%$ do modelo de desdobro tangencial alternado para o modelo de desdobro radial. Isto se deve ao fato deste material ser oriundo de ripas abaixo de $2,50 \mathrm{~m}$ de comprimento, ou seja, todo o material que não dava origem a outro tipo de produto era utilizado para produzir peças com comprimentos variados, agregando valor à madeira que poderia ser rejeitada.

Para a madeira de caibro ocorreu aumento de 4,38\% do desdobro radial em relação ao desdobro tangencial alternado, o qual é explicado pelo aumento do número de peças do segundo e sexto corte da serra de fita vertical deste modelo (Figura 1B), ou seja, as pranchas que não apresentavam largura para originar vigamento eram destinadas para obtenção de caibros.

Destes cortes citados também se aproveitavam as peças com menor espessura para obtenção de madeira para o mercado moveleiro, porém como pode ser observado, houve decréscimo deste produto de $10,62 \%$ do desdobro tangencial alternado para o desdobro radial, justamente por ocorrer maior aproveitamento desta parte da madeira para a confecção de caibros.

De fato é necessária a separação de toras por classes diamétricas e definição de modelos de desdobro adequados para atingir coeficientes de rendimentos elevados para determinados produtos desejados, o que resulta em aumento na lucratividade e rentabilidade da empresa.

\section{Conclusões}

A baixa conicidade obtida evidência que as toras utilizadas apresentaram características adequadas para uso em serraria, proporcionando rendimentos satisfatórios.

A classe diamétrica e o modelo de desdobro influenciam diretamente no rendimento de madeira serrada, nos tipos e quantidades de produtos gerados.

Os rendimentos médios obtidos para a madeira serrada de Eucalyptus ssp. são considerados altos, sendo o rendimento médio para o modelo de desdobro radial maior que o rendimento médio para modelo de desdobro tangencial alternado.

\section{Agradecimentos}

Ao Conselho Nacional de Desenvolvimento Científico e Tecnológico e a empresa Mademape Indústria Madeireira Ltda. 


\section{YIELD AND VARIETY OF PRODUCTS IN THE SAWING OF LOGS Eucalyptus spp.}

ABSTRACT: The study was carried out in a sawmill with the objective of evaluating the conicity, yield, variety and number of products from the cutting of sawed Eucalyptus logs through two cutting templates, in two diametric grades. Logs of Eucalyptus spp. were separated into a mean diameter class of $33.0 \mathrm{~cm}$ and 47.0 $\mathrm{cm}$. For logs of the smaller diameter class, the alternating tangential cutting template was applied and for larger diameter $\operatorname{logs}$, the radial cutting template. From the processing of logs, the obtained products were classified and quantified, obtaining the yield of each cutting model. As for the conicity parameter, the results represented good quality logs, varying from $0.92 \mathrm{~cm} / \mathrm{m}$ in logs of smaller diameter to $1.42 \mathrm{~cm} / \mathrm{m}$ in logs of larger diameter. The radial cutting template, in addition to presenting a higher yield $(53.43 \%)$ than the alternating tangential cutting $(47.08 \%)$, showed a higher percentage of the company's main product (beam). The choice of the appropriate cutting template for each diameter class is a suitable method to reduce losses and maximize yields in sawmills.

Keywords: Lumber. Profitability. Sustainability. Quality.

\section{Referências}

[1] MÜLlER, B. V.; ROCHA, M. P. da; CUNHA, A. B.; KLITZKE, R. J.; NICOLETTI, M. F.; Avaliação das principais propriedades físicas e mecânicas da madeira de Eucalyptus benthamii Maiden et Cambage. Floram, 21 (4): 535-542. 2014. dx.doi.org/10.1590/2179-8087.050413

[2] FERREIRA, S.; LIMA, J. T.; ROSADO, S. C. S.; TRUGILHO, P. F.; Influência de métodos de desdobro tangencial no rendimento e na qualidade da madeira de clones de Eucalyptus spp. Cerne, Vol. 10, n. 1, p. 10-21, 2004.

[3] VALÉRIO, A. F.; WATZLAWICK, L. F.; SANTOS, R. T. dos; BRANDELEIRO, C.; KHOELER, H. S.; Quantificação de resíduos e rendimento no desdobro de Araucaria angustifolia (BERTOL.) O. KUNTZE. Floresta, Curitiba, Vol. 37, n. 3, p. 387-398, 2007.

[4] JUIZO, C. G. F.; ROCHA, M. P.; BILA N. F. B.; Avaliação do rendimento em madeira serrada de eucalipto para dois modelos de desdobro numa serraria portátil. Floresta e Ambiente, Vol. 21, n. 4, p. 543-550, 2014. doi: 10.1590/21798087.062213.

[5] VITAL, B. R.; Planejamento e operação de serrarias. Viçosa, MG: UFV. 2008.

[6] SCANAVACA, Jr. L.; GARCIA, J. N.; Rendimento em madeira serrada de Eucalyptus urophylla. Scientia Forestalis, Vol. 63, n. 1, p. 32-43, 2003.

[7] ROCHA, M. P. da; Técnicas de planejamento em serrarias. Série Didática FUPEF, Curitiba, n. 02/01, 121 p, 2002.

[8] HUSCH, B.; MILLER, C. I.; BEERS, T. W.; Forest mensuration. New York: Ronald Press Co., 410 p, 1982.
[9] GONÇALVES, F. G.; OLIVEIRA, T. S.; SIVA, G.F.; NAPPO, M. E.; FILHO, M. T.; Parâmetros dendrométricos e correlações com propriedades tecnológicas em um híbrido clonal de Eucalyptus urophylla x Eucalyptus grandis. Árvore, Vol. 34, n. 5, p. 947-959, 2010. doi.org/10.1590/S0100-67622010000500020

[10] Instituto Brasileiro de Desenvolvimento Florestal - IBDF Norma para medição e classificação de toras de madeira de folhosas. Brasília. 42 p, 1984.

[11] DEL MENEZZI, C. H. S.; NAHUZ, M. A. R.; SOUZA, MR de.; Aspectos Tecnológicos na produção de madeira serrada de Eucalyptus cloeziana F. Muell. Brasil Florestal, Vol. 70, p. 75-82, 2001.

[12] LOPES, M. C.; HASELEIN, C. R.; SANTINI, E. J.; LONGHI, S. J.; ROSSO, S.; FERNANDES, D. L. G.; MENEZES, L. F.; Agrupamento de árvores matrizes de Eucalyptus grandis em função das variáveis dendrométricas e das características tecnológicas da madeira. Ciência Florestal, Vol. 14, n. 2, p. 133-144, 2004.

[13] ROCHA, M. P. da; Eucalyptus grandis Hill ex Maiden e Eucalyptus dunnii Maiden como fontes de matéria prima para serrarias. Tese (Doutorado em Ciências Florestais) Setor de Ciências Agrárias, Universidade Federal do Paraná. 186 p, 2000.

[14] VIANNA NETO, J. A.; Considerações básicas sobre o desdobro de Pinus spp. Silvicultura, Curitiba, Ano IX. 34: p. 15-19, 1984.

[15] SANTOS, I. S.; LIMA, J. T.; SILVA, J. R. M. da; Avaliação de pisos produzidos a partir de toras de clones de Eucalyptus sp. com pequenos diâmetros. Cerne, Vol. 16, n. 4, p. 473-478, 2010. dx.doi.org/10.1590/S010477602010000400007.

[16] BIASI, C. P.; ROCHA, M. P.; Rendimento em madeira serrada e quantificação de resíduos para três espécies tropicais. Floresta, Vol. 37, n. 1, p. 95-108, 2007.

[17] MELO, R. R.; ROCHA, M. J.; JUNIOR, F. R.; STANGERLIN, D. M.; Análise da influência do diâmetro no rendimento em madeira serrada de cambará. Pesquisa Florestal Brasileira, Vol. 36, n. 88, p. 393-398, 2016. doi: 10.4336/2016.pfb.36.88.1151.

[18] GARCIA, F. M.; Rendimento operacional de uma serraria com a espécie cambará (Qualea albiflora warm.) na região amazônica. 2013, 83p. Dissertação (Mestre em Ciência Florestal) - Universidade Estadual Paulista "Júlio de Mesquita Filho", Botucatu.

[19] MONTEIRO, T. C.; LIMA, J.T.; SILVA, J. R. M.; TRUGILHO, P.F.; ANDRADE, B. C. L.; Avaliação do desdobro de toras de Eucalyptus para a obtenção de peças estruturais. Cerne, Vol. 19, n. 3, p. 357-364, 2013. 